University of Wollongong

Research Online

Faculty of Engineering and Information

Faculty of Engineering and Information

Sciences - Papers: Part B

Sciences

2018

Sonication-enabled rapid production of stable liquid metal nanoparticles grafted with poly(1- octadecene-alt-maleic anhydride) in aqueous solutions

Yiliang Lin

North Carolina State University

Jan Genzer

North Carolina State University

Weihua Li

University of Wollongong, weihuali@uow.edu.au

Ruirui Qiao

Monash University

Michael Dickey

North Carolina State University

See next page for additional authors

Follow this and additional works at: https://ro.uow.edu.au/eispapers1

Part of the Engineering Commons, and the Science and Technology Studies Commons

Research Online is the open access institutional repository for the University of Wollongong. For further information contact the UOW Library: research-pubs@uow.edu.au 


\title{
Sonication-enabled rapid production of stable liquid metal nanoparticles grafted with poly(1- octadecene-alt-maleic anhydride) in aqueous solutions
}

\begin{abstract}
Gallium-based liquid metals are attractive due to their unique combination of metallic and fluidic properties. Liquid metal nanoparticles (LM NPs), produced readily using sonication, find use in soft electronics, drug delivery, and other applications. However, LM NPs in aqueous solutions tend to oxidize and precipitate over time, which hinders their utility in systems that require long-term stability. Here, we introduce a facile route to rapidly produce an aqueous suspension of stable LM NPs within five minutes. We accomplish this by dissolving poly(1-octadecene-alt-maleic anhydride) (POMA) in toluene and mixing with deionized water in the presence of a liquid metal (LM). Sonicating the mixture results in the formation of toluene-POMA emulsions that embed the LM NPs; as the toluene evaporates, POMA coats the particles. Due to the POMA hydrophobic coating, the LM NPs remain stable in biological buffers for at least 60 days without noticeable oxidation, as confirmed by dynamic light scattering and transmission electron microscopy. Further stabilization is achieved by tuning the LM composition. This paper elucidates the stabilization mechanisms. The stable LM NPs possess the potential to advance the use of LM in biomedical applications.
\end{abstract}

\section{Keywords}

anhydride), octadecene-alt-maleic, poly(1-, grafted, nanoparticles, aqueous, metal, solutions, liquid, stable, production, rapid, sonication-enabled

\section{Disciplines \\ Engineering | Science and Technology Studies}

\section{Publication Details}

Lin, Y., Genzer, J., Li, W., Qiao, R., Dickey, M. D. \& Tang, S. (2018). Sonication-enabled rapid production of stable liquid metal nanoparticles grafted with poly(1- octadecene-alt-maleic anhydride) in aqueous solutions. Nanoscale, 10 (42), 19871-19878.

\section{Authors}

Yiliang Lin, Jan Genzer, Weihua Li, Ruirui Qiao, Michael Dickey, and Shiyang Tang 


\section{Sonication-enabled Rapid Production of Stable Liquid Metal}

\section{Nanoparticles Grafted with Poly(1-octadecene-alt-maleic anhydride) in}

\section{Aqueous Solutions}

Yiliang Lin ${ }^{\mathrm{a}}$, Jan Genzer ${ }^{\mathrm{a}}$, Weihua Li ${ }^{\mathrm{b}}$, Ruirui Qiao ${ }^{\mathrm{c}}$, Michael D. Dickey*a, Shi-Yang Tang*b

a Department of Chemical and Biomolecular Engineering, North Carolina State University, Raleigh, NC, USA.

Email: $\underline{\text { mddickey@ncsu.edu }}$

${ }^{\mathrm{b} S} \mathrm{School}$ of Mechanical, Materials, Mechatronic and Biomedical Engineering, University of Wollongong, Australia

E-mail: shiyang@uow.edu.au

${ }^{\mathrm{c}} \mathrm{ARC}$ Centre of Excellence in Convergent Bio-Nano Science and Technology, Drug Delivery, Disposition and Dynamics Theme, Monash Institute of Pharmaceutical Sciences, Monash University, Parkville, VIC 3052, Australia

Keywords: Liquid metal; EGaIn; Nanoparticles; Functional material; Sonication 


\begin{abstract}
Gallium-based liquid metals are attractive due to their unique combination of metallic and fluidic properties. Liquid metal nanoparticles (LM NPs), produced readily using sonication, find use in soft electronics, drug delivery, and other applications. However, LM NPs in aqueous solutions tend to oxidize and precipitate over time, which hinders their utility in systems that require long-term stability. Here we introduce a facile way to rapidly produce aqueous suspension of stable LM NPs within five minutes. We accomplish this by dissolving poly(1-octadecene-alt-maleic anhydride) (POMA) in toluene and mix within deionized water in the presence of liquid metal (LM). Sonicating the mixture results in the formation of toluene-POMA emulsions that embed the LM NPs; as the toluene evaporates, POMA coats the particles. Due to the POMA hydrophobic coating, the LM NPs remain stable in biological buffer for at least 60 days without noticeable oxidation, as confirmed by dynamic light scattering and transmission electron microscopy. The stabilization is further achieved by tuning the LM composition. This paper elucidates the stabilization mechanisms. The stable LM NPs possess the potential to advance the use of LM in biomedical applications.
\end{abstract}




\section{Introduction}

Metals that exist as liquids at room temperature are attractive due to their unique combination of metallic and fluidics properties. As fluids, liquid metals are the softest among all conductive materials ${ }^{1}$. Gallium or its alloys are promising liquid metal candidates due to their low toxicity and negligible vapor pressure ${ }^{2}$. Multiple methods $\mathrm{s}^{3,4}$ have been developed to pattern gallium-based liquid metal and a variety of applications, including, but not limited to, soft electronics ${ }^{5,6}$, microfluidics $^{7}$, composites $^{8}$ and motors 9 .

While most conventional metallic nanoparticles (NPs) are synthesized through a tedious bottomup approach (i.e., reducing precursor metal salts), the fluidic nature of liquid metals (LMs) enables facile methods to produce LM NPs. Disruptive forces induced by acoustic stimulation ${ }^{10-15}$, microfluidic devices ${ }^{16,17}$ or shearing ${ }^{18}$ have been utilized to break the LMs into nanosized particles. Spontaneous formation of an oxide skin on the surface of the metal helps to stabilize the resulting LM NPs. The oxide skin of gallium-based is composed primarily of gallium oxide ${ }^{19,20}$ and the growth of the oxide could be controlled either through thiolation ${ }^{21}$ or thermal oxidation ${ }^{22}$. While the thiolation mitigates (but not eliminates) the growth of the oxide skin, in thermal oxidation, the oxide skin grows thicker with increasing temperature, as expected. Interestingly, the texture and roughness of the oxide skin can be manipulated through thermal oxidation in a temperature-dependent manner ${ }^{22}$. LM NPs are soft and conductive, and thus suitable for inkjet printing and also soft electronics applications, i.e., soft circuit components ${ }^{23-26}$ or antennas ${ }^{27}$. The presence of the oxide skin also endows the formation of $\mathrm{LM} /$ metal oxide framework, which was utilized for photocatalytic applications ${ }^{28,29}$. More interestingly, the morphology of LM NPs is transformable under $\mathrm{pH}$ or light stimulus, and several studies have demonstrated the possibility to use LM NPs for drug delivery, specifically for tumour treatment ${ }^{30-32}$. A recent review summarizes the state-of-art biomedical applications involving liquid metals, 
demonstrating the low-toxicity of liquid metals for bio-applications ${ }^{33}$. In addition, LM NPs also find usage in room-temperature sintering ${ }^{34}$, batteries $^{35}$ and energy harvesting ${ }^{36}$.

Sonication of LM in ethanol produces stable LM NPs. However, sonicating LM in water results in unstable suspensions (i.e., the LM NPs eventually settle due to gravity). Surfactants could be utilized to slow down the precipitation, yet, in the presence of oxygen and water, LM NPs will transform into $\mathrm{GaOOH}$ nanorods due to the oxidation of gallium ${ }^{37}$. Therefore, it is desirable to have a coating that can both stabilizes the LM NPs and prevents the oxidation reaction on the LM NPs surface. With the presence of a hydrophilic polymer coating, such as polymeric grafts made of brushed polyethylene glycol (bPEG) ${ }^{17}$, the LM NPs could remain stable in aqueous environments up to a few days. Unfortunately, the LM NPs will still precipitate in biological buffers, which hinders their use in biomedical applications. Here, we demonstrate that it is possible to synthesize LM NPs in buffer solution that remain stable at least 60 days by grafting a hydrophobic polymer layer comprising poly(1octadecene-alt-maleic anhydride) (POMA) on the surface of the LM NP. This is enabled by dissolving hydrophobic POMA into toluene to form emulsions within aqueous phase that can encapsulate LM NPs, and toluene subsequently evaporates upon sonication. It takes only 5 minutes to produce stable LM NPs in a one-pot synthesis. We further demonstrate that the stabilization could be achieved by tuning the composition of the LM alloy. The stabilization mechanism is studied and elucidated systematically in this work.

\section{Experimental}

Chemical preparation: EGaIn liquid metal, toluene, chloroauric acid $\left(\mathrm{HAuCl}_{4}\right)$, poly $(1$-octadecenealt-maleic anhydride) (POMA, $\mathrm{M}_{\mathrm{n}} \sim 30-50 \mathrm{kDa}$ ), poly(methyl vinyl ether-alt-maleic anhydride) (PMVEMA, $\mathrm{M}_{\mathrm{n}}=216 \mathrm{kDa}$ ), and poly(styrene-co-maleic anhydride) (PSMA, $\mathrm{M}_{\mathrm{n}}=224 \mathrm{kDa}$ ), were 
purchase from Sigma Aldrich, USA. $\mathrm{Ga}_{20} \mathrm{In}_{80}$ alloy was prepared by melting and mixing $16 \mathrm{~g}$ of indium and $4 \mathrm{~g}$ of gallium at $100{ }^{\circ} \mathrm{C}$. We used a probe sonication system (QSONICA, Q700, 40\% amplitude) to break the alloys into NPs.

Characterization: Scanning electron microscope (SEM) images were obtained using a JEOL JSM7500FA scanning electron microscope. Transmission electron microscope (TEM) images and energydispersive X-ray spectroscopy (EDS) maps were obtained using FEI Titan 80-300 and JEOL JEMARM200f transmission electron microscopes. A zeta-sizer (Zetasizer Nano ZS, Marvern Instrument, USA) was used to measure the size distribution and zeta potential of the LM NPs. The concentration of the EGaIn nanoparticles was measured by weighing the dried suspensions.

\section{Results and discussion}

Figure 1a shows a schematic of the proposed method leading to the one-step production of stable and functionalizable EGaIn NPs with a hydrophobic insulating coating layer in an aqueous solution. In a typical experiment, we dissolve $50 \mathrm{mg}$ POMA powder into $1 \mathrm{~mL}$ toluene and add $100 \mu \mathrm{L}$ of the POMA-toluene solution into an $8 \mathrm{~mL}$ glass vial, which contains $5 \mathrm{~mL}$ deionized (DI) water (final POMA concentration in water is $1 \mathrm{mg} / \mathrm{mL}$ ) and a $50 \mu \mathrm{L}$ of EGaIn. Toluene dissolves POMA, which is otherwise insoluble in water. Next, we sonicate the mixture using a probe sonication system at $40 \%$ amplitude for $300 \mathrm{~s}$. Upon the activation of the probe, EGaIn breaks into NPs. In addition, the sonication should form a POMA-toluene emulsion (cf. Fig. 1a). The POMA macromolecules act as emulsifiers where the maleic anhydride groups are hydrolysed into carboxylic acid groups that interact with EGaIn NPs, while the hydrophobic octadecyl (C18) hydrocarbon chains prevent EGaIn NPs from the aggregation and oxidation. Meanwhile, sonication also enables the POMA coated EGaIn NP to interact with the toluene via the C18 chains and thereby become encapsulated within the emulsion (cf. 
Fig. 1a). In the end, toluene evaporates upon heating caused by prolonged sonication (cf. Fig. 1a). This enables the formation of double POMA-layer grafted EGaIn NPs in water. The interaction between POMA and EGaIn likely occurs through hydrogen bonding. Specifically, POMA hydrolysed in aqueous solution forms carboxyl groups, which hydrogen bond with the native gallium oxide on the surface of EGaIn. We performed time-of-flight secondary ion mass spectrometry (ToF-SIMS) experiments on EGaIn-POMA samples and detected signals from $\mathrm{GaO}^{-}$and $\mathrm{COO}^{-}$. However, ToFSIMS spectra (not shown) contain no evidence for the formation of complexes between carboxyl groups in POMA and gallium (or gallium oxide), which indicates that gallium is not likely covalently attached to the carboxyl groups.

Figure $1 \mathrm{~b}$ shows the size distribution of the POMA-grafted EGaIn NPs measured using a zetasizer, where the peak size is centered at $\sim 170 \mathrm{~nm}$. The NP suspension is very stable; the dark color indicates high concentration of the NP $(\sim 2 \mathrm{mg} / \mathrm{mL})$, as shown in the inset of Fig. 1b. The concentration of the LM NPs could be further increased to $8 \mathrm{mg} / \mathrm{mL}$ following another sonication cycle. To the best of our knowledge, this is the highest concentration of LM NPs in aqueous system reported to date. Fig. 1c shows the TEM images of the LM NPs. The zoomed-in image in Fig. 1c shows the thin layer of oxide $(\sim 2.5 \mathrm{~nm})$ formed on the LM NP surface No diffraction pattern was observed from the convergent-beam electron diffraction (CBED) measurement (see Fig. S1 in ESI), indicating that NPs remains in a liquid state. We further obtained EDS maps to examine the distribution of gallium, indium, and oxygen within the EGaIn NPs, as shown in Fig. 1d. The EDS mapping indicates a uniform distribution of gallium and indium within the NPs; the presence of oxygen is attributed to the oxide layer and the POMA polymer coating. Since we obtained the TEM image using a copper grid coated with an evaporated carbon film, the POMA polymer coating cannot be distinguished from the carbon film background due to the lack of contrast. Therefore, we obtained additional TEM images using a grid coated with a lacey carbon film to avoid background (cf. Fig. S2a), from which we discovered that the thickness of the POMA coating is $\sim 6$ to $7 \mathrm{~nm}$. We also prepared LM NPs grafted with small 
molecules of trisodium citrate by sonicating $50 \mu \mathrm{L}$ of EGaIn within $5 \mathrm{~mL}$ trisodium citrate $(1 \mathrm{mM})$ solution for $5 \mathrm{~min}$, and we found that the hydrodynamic size distribution of the NPs becomes narrower and smaller; the peak size is found at $\sim 150 \mathrm{~nm}$ (cf. Fig. S2b). This result is in line with the TEM measurements where the increase of the particle size is due to the POMA polymer coating.

We also measured the zeta potentials for the NPs with or without the POMA grafted layer. The zeta potential of EGaIn NPs without POMA grafting is low $(-6.1 \pm 5.1 \mathrm{mV})$ in water and the NPs aggregate rapidly after sonication. On the contrary, we obtained a large negative zeta potential for POMA grafted EGaIn NPs $(-47.9 \pm 9.2 \mathrm{mV})$, confirming hydrolysis and charging of $\mathrm{COO}^{-}$originating from maleic anhydride units on the grafted POMA coatings. This indicates that the stabilization of LM NPs can be attributed (at least in part) to electrostatic repulsion among $\mathrm{COO}^{-}$. we note that steric effects due to the $\mathrm{C} 18$ units might also play a role in stabilization. To better understand the production mechanism depicted in Fig. 1a, we further conducted a series of proof-of-concept experiments to validate our proposed scheme, as discussed in the following sections. 


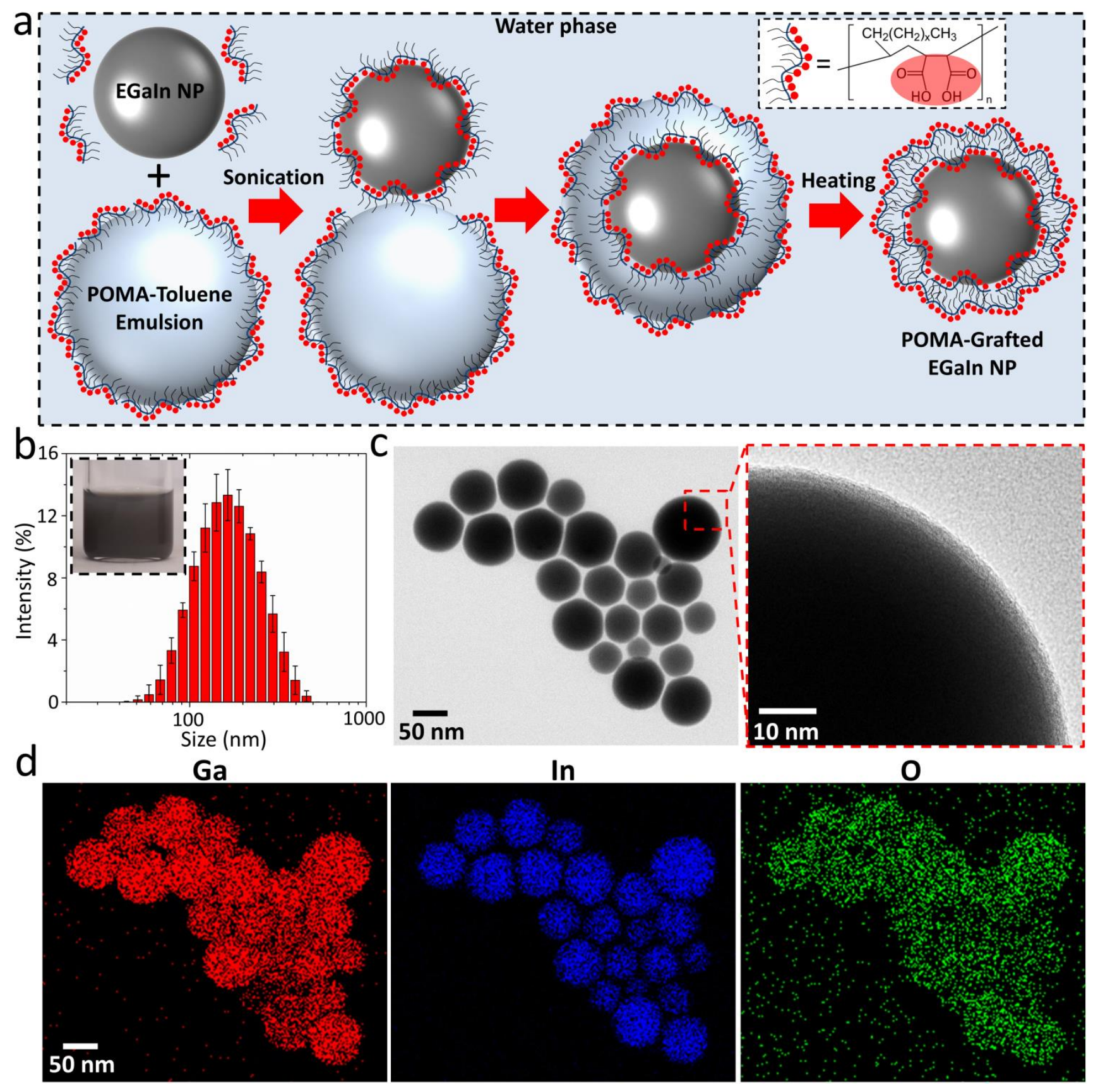

Figure 1. Production of POMA grafted EGaIn NPs. (a) Schematic depicting the mechanism leading to the production of stable liquid metal NPs. (b) Hydrodynamic size distribution of the POMA grafted EGaIn NPs; the inset shows the NP colloidal suspension. (c) TEM images of the produced NPs. (d) EDS mapping of gallium, indium, and oxygen for the NPs.

Figure 2a presents sequential snapshots taken during the production of EGaIn NPs. We observed the formation of a white-colored suspension after activating the probe for $10 \mathrm{~s}$. We attribute this suspension to the formation of POMA-toluene emulsions ( $c f$. the emulsion also forms without LM in Fig. 2b). The 
temperature of the suspension increases to $\sim 95{ }^{\circ} \mathrm{C}$ after sonicating for $300 \mathrm{~s}$, as measured by a thermocouple. The color of the suspension changes gradually to dark grey after activating the probe for $300 \mathrm{~s}$, indicating the evaporation of toluene and the formation of POMA-grafted LM NPs. A series of control experiments shows that no stable LM NP suspensions are obtained for the cases of 1) sonicating EGaIn in water; 2) sonicating EGaIn in water and POMA powder; and 3) sonicating EGaIn in water and toluene (see Fig. S3 in ESI for details). This indicates that the presence of POMA-toluene emulsions is crucial for producing stable POMA-grafted LM NPs. The formation of POMA-toluene emulations is evidenced by sonicating water-POMA-toluene mixture without the presence of EGaIn, as shown in Fig. 2b. We can clearly see the white-color suspension after activating the sonication probe for $120 \mathrm{~s}$; the liquid gradually becomes clearer after prolonging the sonicating time to $300 \mathrm{~s}$, indicating gradual evaporation of toluene induced by the heat. Similarly, Fig. 2c shows the centrifuged (at 9600 $\mathrm{g}$ for $5 \mathrm{~min}$ ) NP suspensions with sonication time of 120 s and 300 s respectively, in which the supernatant is opaque with a thick layer of toluene-EGaIn mix at $120 \mathrm{~s}$, while the supernatant is transparent at $300 \mathrm{~s}$.

We further conducted a series of experiments to demonstrate the importance of forming POMAtoluene emulsions at the beginning of the production process for achieving stable POMA grafted EGaIn NPs. Fig. 2d shows the supernatant of the LM NP suspensions obtained by pre-sonicating the water-POMA-toluene mixture for 0, 10, 60, 120, and $300 \mathrm{~s}$ before adding EGaIn for additional $300 \mathrm{~s}$ sonication. The concentration of the LM NP reduces significantly by prolonging the pre-sonication time. This may be attributed to the fact that the evaporation of toluene prevents the POMA-toluene emulsions to interact with and encapsulate POMA-coated EGaIn NPs (cf. Fig. 1a), and consequently, no stable double-layer POMA grafted LM NPs can be produced.

We also performed galvanic replacement for the surface of EGaIn NPs with gold (Au) to prove the existence of the hydrophobic layer formed between the two layers of POMA grafting molecules 
on a LM NP. In doing so, we added $10 \mu \mathrm{L} \mathrm{HAuCl}_{4}$ solution (concentration of $10 \mathrm{mM}$ ) into $1 \mathrm{~mL}$ of the obtained EGaIn NP suspension and gently mixed it for $5 \mathrm{~min}$. The straightforward reaction reduces $\mathrm{Au}^{+}$ions to metallic $\mathrm{Au}-\mathrm{NPs}$ on the surface of EGaIn (the other half reaction involves Ga oxidation). We compared the corresponding TEM images and EDS mappings obtained for the galvanic replacement experiments conducted using POMA grafted NPs with $120 \mathrm{~s}$ (Fig. 2e) and $300 \mathrm{~s}$ (Fig. 2f) sonication time, as well as bPEG (Mn $20 \mathrm{kDa}$, see details in previous work ${ }^{17}$ and Fig. S4 in ESI) grafted EGaIn NPs (Fig. 2g). The surfaces of the POMA-grafted NPs contain fewer AuNPs, and the size of the formed AuNPs is relatively large in comparison to the case of bPEG-grafted NPs. We have previously shown that the surface of bPEG-grafted EGaIn NPs can be uniformly coated with silver NPs after galvanic replacement due to the uniform reaction occurred on the surface of EGaIn ${ }^{17}$. However, for the case of POMA grafted NPs, the existence of a hydrophobic layer can prevent the reaction. We reason that the larger size of the AuNPs observed is due to the continuous growth of a particle from a localized point on the surface of EGaIn with defects in POMA coating. Furthermore, there is likely additional unevaporated toluene present in the hydrophobic layer for the NPs obtained with $120 \mathrm{~s}$ sonicating time, and this layer further prevents the galvanic replacement reaction, therefore, reducing the number of Au-NPs formed on the surface (see Fig. 2e and Fig. 2f).

Such a hydrophobic insulating layer prevents the hydrolysis and oxidation of the EGaIn NPs. This is evidenced by control experiments conducted using PMVEMA and PSMA, as the grafting macromolecules. We observed the formation of a relatively thick (10-20 nm) gallium oxide shell on the NPs and gallium oxide nanodisks only 5 days after production particle, as shown in Fig. $2 \mathrm{~h}$ (see also Fig. S5 in ESI), while no change for the POMA grafted NPs was observed in water even 60 days after production, as shown in Fig. 2i. The hydrophobic insulating layer can also prevent dissolution of the NPs from etchant, such as strong acid, as shown in Fig. S6. The developed simple and straightforward NP grafting method by sonicating NPs and POMA-toluene solution can also be applied 
ESI.

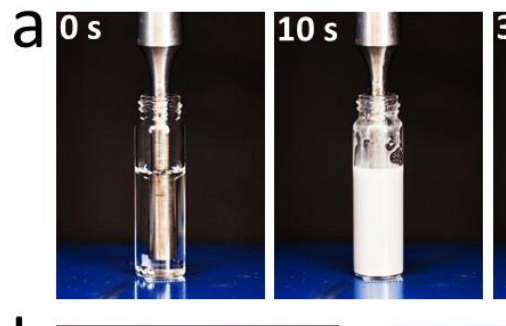

b

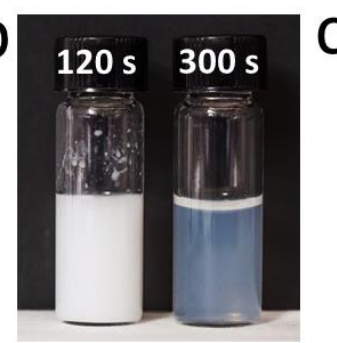

e
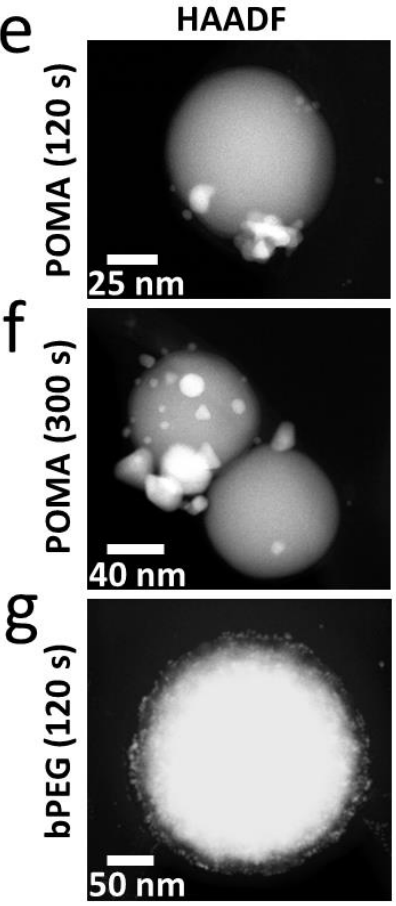
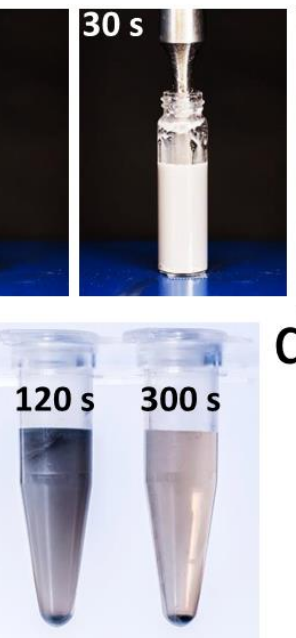

$\mathrm{Ga}$
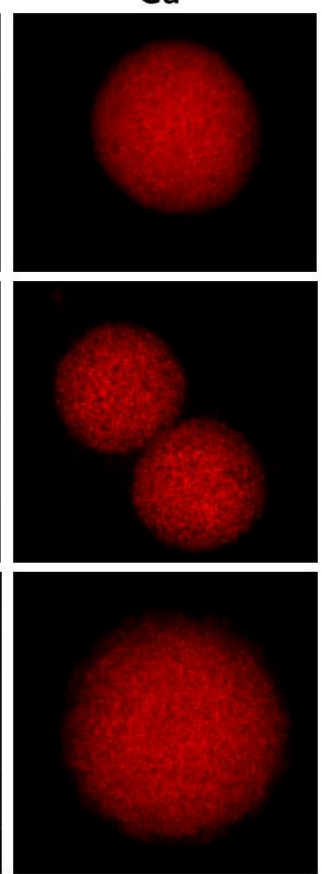
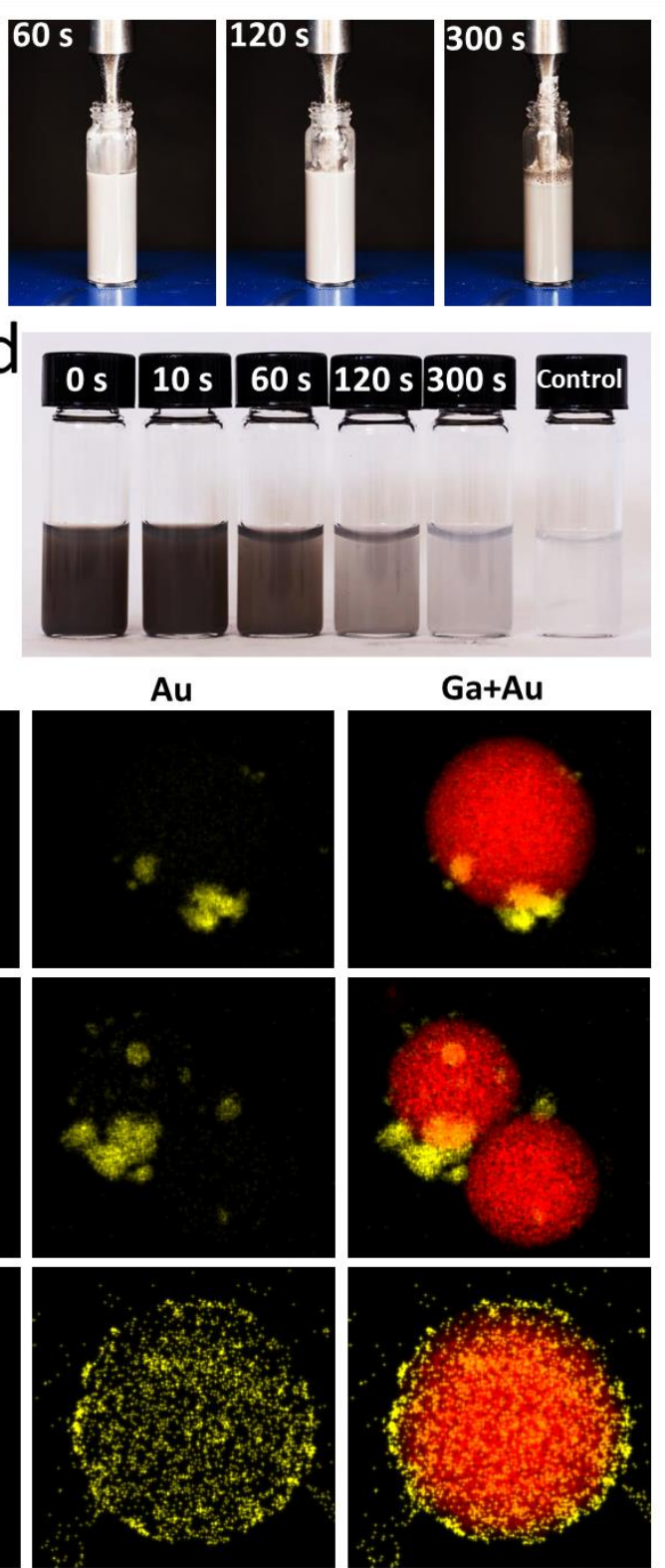
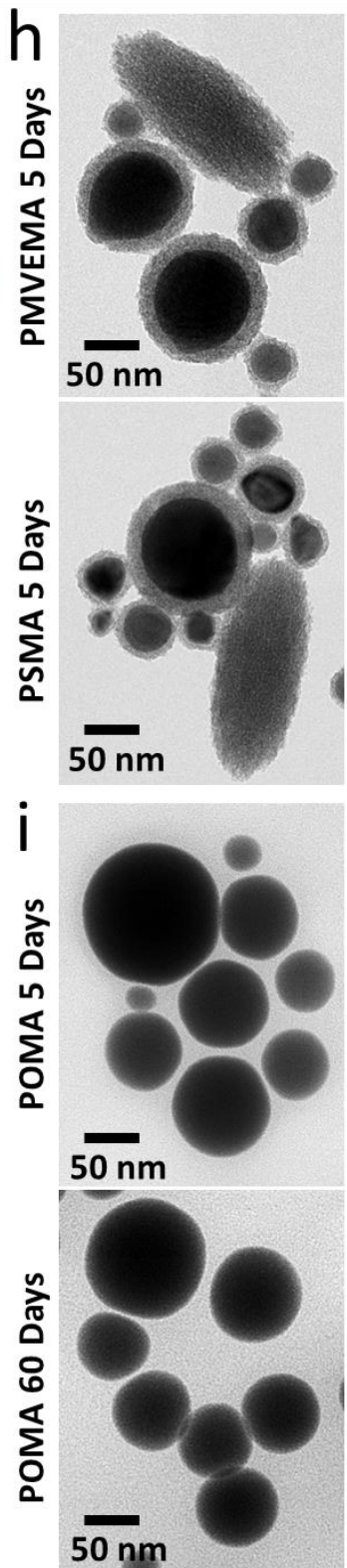

Figure 2. Investigating the mechanism behind the production of POMA grafted EGaIn NPs. (a) Sequential snap shots showing the NP suspension during the production process. (b) Water-POMAtoluene mix 120 and $300 \mathrm{~s}$ after activating the sonication probe. (c) Centrifuged LM NP suspension 120 and $300 \mathrm{~s}$ after activating the sonication probe. (d) Image of the supernatants for LM NPs produced with different pre-sonicating time; the control sample lacks EGaIn during sonication. TEM image and EDS mapping of the POMA grafted EGaIn NPs produced with (e) 120, and (f) 300 s sonicating time after galvanic replacement reaction. (g) TEM image and EDS mapping of the bPEG-grafted EGaIn NPs after galvanic replacement reaction. (h) TEM images for PMVEMA and PSMA grafted LM NPs 5 days after production. (I) TEM images for POMA grafted LM NPs 5 and 60 days after production. 
We demonstrated that the POMA-grafted coatings are able to stabilize the EGaIn NPs in water. We further investigated the long-term stability of such POMA grafted EGaIn NPs within biological buffers containing competitive ions such as phosphate-buffered saline (PBS). We added $100 \mu \mathrm{L}$ of ten times concentrated (10x) PBS buffer into $900 \mu \mathrm{L}$ of the produced EGaIn NP suspension, and Fig. 3a displays the measured size distribution for the NPs over a period of 60 days. The NP suspension is very stable (see Fig. 3a right-corner inset) with only a minor shift of the size distribution towards larger sizes after 60 days. We further examined the EGaIn NPs using TEM, as shown in the inset of Fig. 3a, where we did not observe the formation of gallium oxide nanodisks. The minor shift of the size distribution is likely due to the gradual growth of gallium oxide on the surface of EGaIn in PBS, indicating that oxygen molecules are still able to penetrate the hydrophobic insulating layer. This result is interesting as we initially expected that the ions within PBS could compromise the electrostatic repulsion force between NPs and induce aggregation.

To better understand the mechanism behind the stabilization, we suspended the LM NPs within $0.01 \mathrm{M}$ sodium phosphate buffers with the $\mathrm{pH}$ of 2, 7 and 12 to suppress or facilitate the hydrolysis of the grafted POMA. Fig. 3b shows the size distributions of the NPs 2 days after suspending. The LM NPs are very stable within the buffers with no aggregation observed. We observed that the zeta potential of the NPs reduced significantly to $-19.7 \pm 12.9 \mathrm{mV}$ within the $\mathrm{pH} 2$ buffer, as shown in Fig. 3c. This can be attributed to the suppression of hydrolysis for the grafted POMA at such a low $\mathrm{pH}$, and the absence of NP aggregation indicates that the grafted POMA molecules can provide a barrier to sterically stabilize the NPs. The zeta potential increased to $-44.3 \pm 10.7 \mathrm{mV}$ within the $\mathrm{pH} 7 \mathrm{buffer}$, indicating that the hydrolysis of the grafted POMA is facilitated at a neutral $\mathrm{pH}$ (Fig. 3c), and the LM NPs can be stabilized by the combination of electrostatic repulsion and steric barrier, i.e. electrosterically ${ }^{38}$. The decreased zeta potential within a pH 12 buffer is likely due to the increased ion concentration after adding sodium hydroxide $(\mathrm{NaOH})$ when adjusting the $\mathrm{pH}$. These experiments explain the reason behind the outstanding stability of the NPs within PBS; our measurement shows 
that the zeta potential of the NPs is $-28.9 \pm 3.4 \mathrm{mV}$ within $1 \times \mathrm{PBS}$ (Fig. 3c) and therefore, the existence of the steric barrier formed by POMA facilitates the stabilization. The zeta potential of the NPs further decreased to $-23.9 \pm 2.2 \mathrm{mV}$ and $-16.6 \pm 1.3 \mathrm{mV}$ after we double and triple the concentration of PBS, respectively. Such a high ion concentration eventually induces the precipitation of the NPs, as shown in Fig. S8 in ESI.

Since the POMA grafted LM NPs are stable within biological buffer, we further examined their cytotoxicity on Chinese hamster ovary (CHO) cell line using the Alamar Blue assay (see Fig. S9 for details). Fig. S9 shows the viability of the $\mathrm{CHO}$ cells upon the 24 h exposure to the NPs with different concentrations; we observed that the NPs has no or little effect on the viability of CHO cells for particle concentration less than $62.5 \mu \mathrm{g} / \mathrm{mL}$. 

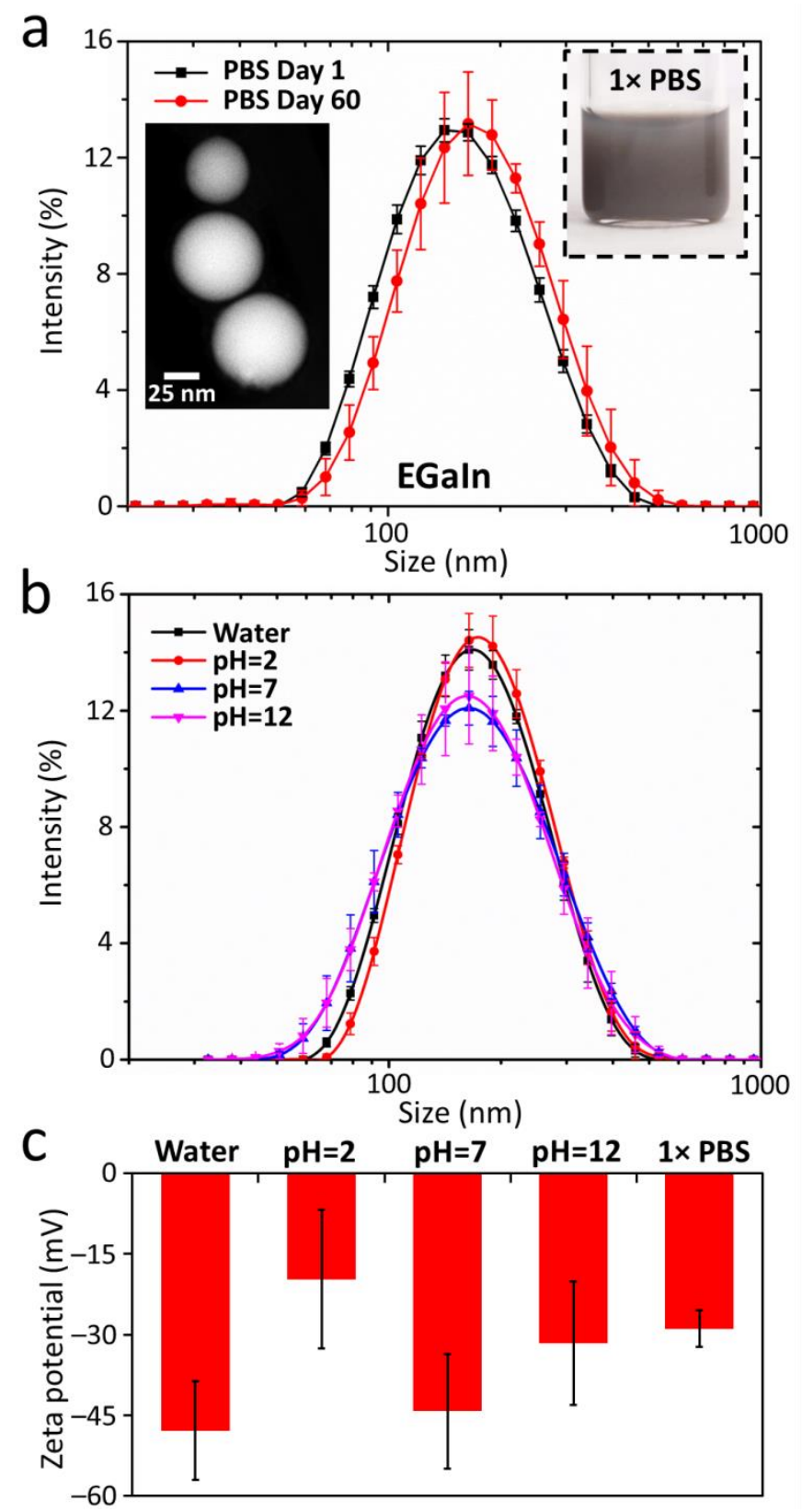

Figure 3. Investigating the stability of POMA grafted liquid metal NPs within PBS buffer. (a) Hydrodynamic size distribution of the POMA grafted EGaIn NPs within 1× PBS buffer over a period of 60 days; the insets shows the NP suspension and TEM image taken 60 days after production. (b) Hydrodynamic size distribution of the LM NPs within water and 0.01 M sodium phosphate buffers with the $\mathrm{pH}$ of 2, 7 and 12. (c) Zeta potential of the POMA-grafted NPs suspended within water, sodium phosphate buffers, and $1 \times$ PBS. 
To minimize the growth of the oxide layer on the EGaIn NP surfaces and to maximize their long-term stability, we utilized an alloy which contains $80 \%$ (by weight) of indium and $20 \%$ of gallium $\left(\mathrm{Ga}_{20} \operatorname{In}_{80}\right)$ for producing the POMA-grafted LM NPs. We hypothesize that increasing the content of indium can minimize the hydrolysis and oxidation of Ga in the NPs. On a related note, it is reported that a much higher concentration of gallium ions is detected than that of indium ions when submerging EGaIn in aqueous solution, indicating the instability of gallium in aqueous system compared to indium ${ }^{39}$. Therefore, it is possible to enhance the stability of gallium-based alloys in aqueous system through decreasing the content of gallium. We also conducted proof-of-concept experiments to compare the chemical stability of EGaIn and $\mathrm{Ga}_{20} \mathrm{In}_{80}$ NPs by sonicating them within DI water for 10 min. Our previously reported results show that EGaIn NPs can be hydrolysed and oxidized to gallium oxide nanodisks 10 min after sonication due to heating ${ }^{37}$. Fig. 4a and Fig. 4b display EDS spectra and SEM images obtained for the EGaIn and $\mathrm{Ga}_{20} \mathrm{In}_{80}$ NPs 10 min after sonication, respectively. The $\mathrm{Ga}_{20} \mathrm{In}_{80}$ alloy is solid at room temperature but can become liquid after heating induced during sonication. Therefore, the bulk material can be broken into LM NPs. We can clearly see that dealloying process occurred for EGaIn NPs and most of the NPs were oxidized to gallium oxide nanodisks (Fig. 4a), while the $\mathrm{Ga}_{20} \mathrm{In}_{80}$ NPs have remained spherical and stable without changes in shape (Fig. 4b). Interestingly, we did not observe diffraction patterns from the convergent-beam electron diffraction (CBED) measurement of the $\mathrm{Ga}_{20} \mathrm{In}_{80}$ NPs, indicating that the NPs of $\mathrm{Ga}_{20} \operatorname{In}_{80}$ alloy are in liquid state. This might be attributed to the supercooling effect of liquid metals ${ }^{40}$. Despite the high content of indium within the NPs, the EDS mappings given in Fig. 4c clearly show the formation of a gallium, instead of indium, oxide layer on the surface of the NPs. During these experiments, we discovered that increasing the indium content can minimize the hydrolysis and oxidation of the NPs within PBS, as evidenced by the measurement of size distribution reported in Fig. 4d, where no shift of the peak was observed within 60 days. We did not observe any changes of the NPs from the TEM images taken 60 days after particle production (Fig. 4d inset). These results demonstrate that this method of producing stable 
liquid metal NPs grafted by POMA represents a significant advance in comparison to previously reported work.
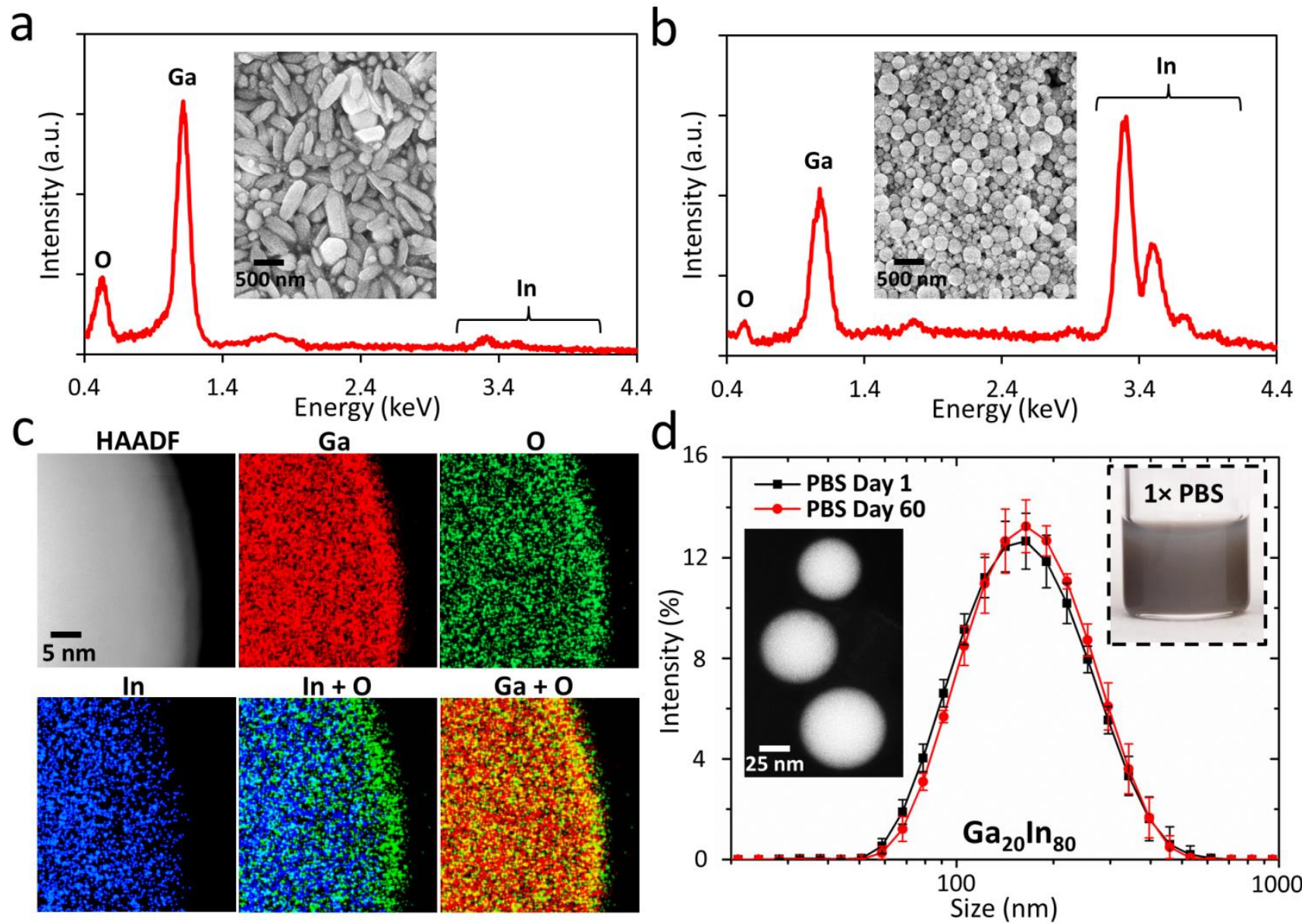

Figure 4. Investigating the enhanced stability of GazoInso liquid metal NPs. EDS spectrum and SEM image for (a) EGaIn, and (b) Ga $\mathrm{G}_{20} \mathrm{In}_{80}$ NPs obtained after 10 min sonication in DI water. (c) EDS mapping of gallium, indium, and oxygen for the $\mathrm{Ga}_{20} \mathrm{In}_{80}$ NPs. (d) hydrodynamic size distribution of the POMA grafted $\mathrm{Ga}_{20} \mathrm{In}_{80}$ NPs within $1 \times$ PBS buffer over a period of 60 days; the insets shows the NP suspension and TEM image taken 60 days after production. 


\section{Conclusions}

In summary, we report on a simple and rapid method for producing stable LM NPs grafted with doublelayered POMA molecules using sonication. It only takes 5 minutes to produce such NPs. The POMA coating not only offers a hydrophobic insulation layer and steric barrier, but also forms a surface with strong negative surface charge that stabilizes the LM NPs. The produced LM NPs are stable in ionic biological buffers, such as PBS, for at least 60 days without aggregation induced by hydrolysis and oxidation. Reducing the gallium content in the alloy further enhances the NP stability. The simplicity and versatility of the production method, along with the excellent stability of the produced LM NPs, has the potential for achieving the further development of LM NP-based biomedical applications.

\section{Conflicts of interest}

There are no conflicts of interest to declare

\section{Acknowledgments}

Dr. Shi-Yang Tang is the recipient of the Vice-Chancellor's Postdoctoral Research Fellowship funded by the University of Wollongong. Dr. Shi-Yang Tang and Dr. Weihua Li acknowledge funding from University of Wollongong University Internationalization Committee (UIC) Grant. Yiliang Lin and Dr. Michael Dickey acknowledge funding from the National Science Foundation through the Research Triangle MRSEC (DMR-1121107). We thank Mr. Steven Zboray for the helpful discussions. The authors acknowledge use of the facilities and the assistance of Dr. David Mitchell at the UOW Electron Microscopy Centre and the use of the Analytical Instrumentation Facility (AIF) at North Carolina State University, which is supported by the State of North Carolina and the National Science Foundation (award number ECCS-1542015).

\section{References}


$1 \quad$ S. Wagner and S. Bauer, MRS Bulletin, 2012, 37, 207-213.

2 W. M. Haynes, CRC Handbook of Chemistry and Physics, 95th Edition, CRC Press, 2014.

3 I. D. Joshipura, H. R. Ayers, C. Majidi and M. D. Dickey, J. Mater. Chem. C, 2015, 3, 3834 3841.

$4 \quad$ M. A. H. Khondoker and D. Sameoto, Smart Mater. Struct., 2016, 25, 093001.

$5 \quad$ M. D. Dickey, Advanced Materials, 2017, 29, 1606425.

6 M. D. Dickey, ACS Appl. Mater. Interfaces, 2014, 6, 18369-18379.

7 K. Khoshmanesh, S.-Y. Tang, J. Yang Zhu, S. Schaefer, A. Mitchell, K. Kalantar-zadeh and M. D. Dickey, Lab on a Chip, 2017, 17, 974-993.

$8 \quad$ N. Kazem, T. Hellebrekers and C. Majidi, Advanced Materials, 2017, 29, 1605985.

9 J. Zhang, Y. Yao, L. Sheng and J. Liu, Advanced Materials, 2015, 27, 2648-2655.

10 J. N. Hohman, M. Kim, G. A. Wadsworth, H. R. Bednar, J. Jiang, M. A. LeThai and P. S. Weiss, Nano Lett., 2011, 11, 5104-5110.

11 J. W. Boley, E. L. White, G. T.-C. Chiu and R. K. Kramer, Adv. Funct. Mater., 2014, 24, 35013507.

12 A. Yamaguchi, Y. Mashima and T. Iyoda, Angew. Chem. Int. Ed., 2015, 54, 12809-12813.

13 W. Zhang, J. Z. Ou, S.-Y. Tang, V. Sivan, D. D. Yao, K. Latham, K. Khoshmanesh, A. Mitchell, A. P. O’Mullane and K. Kalantar-zadeh, Adv. Funct. Mater., 2014, 24, 3799-3807.

14 L. R. Finkenauer, Q. Lu, I. F. Hakem, C. Majidi and M. R. Bockstaller, Langmuir, 2017, 33, 9703-9710.

15 T. R. Lear, S.-H. Hyun, J. W. Boley, E. L. White, D. H. Thompson and R. K. Kramer, Extreme Mechanics Letters, 2017, 13, 126-134.

16 S.-Y. Tang, V. Sivan, P. Petersen, W. Zhang, P. D. Morrison, K. Kalantar-zadeh, A. Mitchell and K. Khoshmanesh, Adv. Funct. Mater., 2014, 24, 5851-5858. 

14, 1800118.

I. D. Tevis, L. B. Newcomb and M. Thuo, Langmuir, 2014, 30, 14308-14313.

M. D. Dickey, R. C. Chiechi, R. J. Larsen, E. A. Weiss, D. A. Weitz and G. M. Whitesides, Adv. Funct. Mater., 2008, 18, 1097-1104.

R. N. S. Sodhi, P. Brodersen, L. Cademartiri, M. M. Thuo and C. A. Nijhuis, Surface and Interface Analysis, 2017, 49, 1309-1315.

Z. J. Farrell and C. Tabor, Langmuir, 2018, 34, 234-240.

J. Cutinho, B. S. Chang, S. Oyola-Reynoso, J. Chen, S. S. Akhter, I. D. Tevis, N. J. Bello, A. Martin, M. C. Foster and M. M. Thuo, ACS Nano, 2018, 12, 4744-4753.

M. G. Mohammed and R. Kramer, Advanced Materials, 2017, 29, 1604965.

M. D. Bartlett, N. Kazem, M. J. Powell-Palm, X. Huang, W. Sun, J. A. Malen and C. Majidi, PNAS, 2017, 114, 2143-2148.

M. D. Bartlett, A. Fassler, N. Kazem, E. J. Markvicka, P. Mandal and C. Majidi, Adv. Mater., 2016, 28, 3726-3731.

26 L. Tang, S. Cheng, L. Zhang, H. Mi, L. Mou, S. Yang, Z. Huang, X. Shi and X. Jiang, iScience, $2018,4,302-311$.

Y. Lin, C. Cooper, M. Wang, J. J. Adams, J. Genzer and M. D. Dickey, Small, 2015, 11, 63976403.

S.-Y. Tang, V. Sivan, K. Khoshmanesh, A. P. O’Mullane, X. Tang, B. Gol, N. Eshtiaghi, F. Lieder, P. Petersen, A. Mitchell and K. Kalantar-zadeh, Nanoscale, 2013, 5, 5949-5957.

X. Tang, S.-Y. Tang, V. Sivan, W. Zhang, A. Mitchell, K. Kalantar-zadeh and K. Khoshmanesh, Appl. Phys. Lett., 2013, 103, 174104.

30 Y. Lu, Q. Hu, Y. Lin, D. B. Pacardo, C. Wang, W. Sun, F. S. Ligler, M. D. Dickey and Z. Gu, Nature Communications, 2015, 6, 10066. 
31 Y. Lu, Y. Lin, Z. Chen, Q. Hu, Y. Liu, S. Yu, W. Gao, M. D. Dickey and Z. Gu, Nano Lett., $2017, \mathbf{1 7}, 2138-2145$.

32 S. A. Chechetka, Y. Yu, X. Zhen, M. Pramanik, K. Pu and E. Miyako, Nature Communications, $2017,8,15432$.

33 J. Yan, Y. Lu, G. Chen, M. Yang and Z. Gu, Chemical Society Reviews, 2018, 23, 2518-2533.

34 S. Çınar, I. D. Tevis, J. Chen and M. Thuo, Scientific Reports, 2016, 6, 21864.

35 Y. Wu, L. Huang, X. Huang, X. Guo, D. Liu, D. Zheng, X. Zhang, R. Ren, D. Qu and J. Chen, Energy \& Environmental Science, 2017, 10, 1854-1861.

36 T. Krupenkin and J. A. Taylor, Nature Communications, 2011, 2, 448.

37 Y. Lin, Y. Liu, J. Genzer and M. D. Dickey, Chem. Sci., 2017, 8, 3832-3837.

38 G. Fritz, V. Schädler, N. Willenbacher and N. J. Wagner, Langmuir, 2002, 18, 6381-6390.

39 J.-H. Kim, S. Kim, J.-H. So, K. Kim and H.-J. Koo, ACS Appl. Mater. Interfaces, 2018, 10, $17448-17454$.

40 M. Losurdo, A. Suvorova, S. Rubanov, K. Hingerl and A. S. Brown, Nature Materials, 2016, 15, 995-1002. 